\title{
SYNTHESIS, THERMAL REACTIVITY, AND ANTIOXIDANT STUDIES OF AMINOGUANIDINIUM SALTS OF ASPARTIC AND GLUTAMIC ACIDS
}

\author{
PARVEEN $S^{1}$, MANIKANDAN D ${ }^{1}$, GOVINDARAJAN $S^{2}$, PERIASAMY PA ${ }^{1}$ \\ ${ }^{1}$ Department of Science and Humanities, Dr. Mahalingam College of Engineering and Technology, Pollachi, Tamil Nadu, India. ${ }^{2}$ Department \\ of Chemistry, Bharathiar University, Coimbatore, Tamil Nadu, India. Email: manikandandhayalan88@gmail.com
}

Received: 29 January 2020, Revised: 18 March 2020, Accepted: 19 March 2020

\section{ABSTRACT}

Objective: Our main scope and objectives are to prepare aminoguanidinium salts of amino acids and to characterize them using analytical, IR, and thermal studies, to study the mode of thermal decomposition of aminoguanidinium salts, and to characterize the antioxidants behavior of aminoguanidinium salts.

Methods: Elemental analysis for $\mathrm{C}, \mathrm{H}$, and $\mathrm{N}$ was performed on a Vario ELIII elemental analyzer. The IR spectra were recorded on a JASCO-4100 spectrophotometer as KBr pellets in the range of $400-4000 \mathrm{~cm}^{-1}$. The simultaneous TG-DTA studies were under taken on a PerkinElmer SII thermal analyzer and the curves obtained in air using platinum cups as holders with $\sim 3 \mathrm{mg}$ of the samples at the heating rate of $10^{\circ} \mathrm{C} / \mathrm{min}$. The antioxidant capacities of different salts were estimated according to the literature procedure.

Results: Aspartic acid forms bis-aminoguanidinium salt, whereas glutamic acid forms both mono- and bis-aminogunidinium salts. The IR spectral data of the aminogunidinium salts of aforesaid acids show N-N stretching frequencies in the region $1110-1202 \mathrm{~cm}^{-1}$ revealing the presence of aminoguanidinium moiety.

Conclusion: The antioxidant properties of these salts were studied using ferric reducing antioxidant power and phosphomolybdenum assay. Results showed significant ferric reducing power which indicated the hydrogen-donating ability of the extract.

Keywords: Aminoguanidine, Amino acids, Antioxidant, Thermal activity.

(C) 2020 The Authors. Published by Innovare Academic Sciences Pvt Ltd. This is an open access article under the CC BY license (http://creativecommons. org/licenses/by/4. 0/) DOI: http://dx.doi.org/10.22159/ajpcr.2020.v13i6.36977

\section{INTRODUCTION}

Recently, there has been a surge in research on the potential role of antioxidants in the treatment of atherosclerosis, heart failure, liver dysfunction, neurodegenerative disorders, cancer, and diabetes mellitus. The main characteristic of an antioxidant is its ability to trap free radicals. Antioxidants provide protection to living organisms from damage caused by uncontrolled production of ROS and concomitant lipid peroxidation, protein damage, and DNA stand breaking. Antioxidant action involves suppressing the formation of ROS, scavenging the free radicals, and reducing agents and quenchers of singlet oxygen formation. The use of synthetic antioxidants must be under strict regulation due to potential health hazards. The search for new antioxidants as alternatives is, therefore, of great interest among researchers.

An amino acid is a type of organic acid that contains an acid functional group and an amine functional group on adjacent carbon atoms. Aspartic acid $\left(\mathrm{H}_{2} \mathrm{Asp}\right)$ is one of two acidic amino acids. Aspartic acid plays an important role as general acids in enzyme active centers, as well as in maintaining the solubility and ionic character of proteins. Aspartic acid crystallized separately as $d$ and $l$ enantiomorphs under an ambient temperature [1]. Aspartic acid undergoes racemic transition at ambient conditions, which found application in chirel fields [2] parity non-conservation, chiral symmetry breaking [3], and cocrystal engineering [4]. The crystal structures of L-aspartic acid [5], DL-aspartic acid [6], DL-aspartic acid nitrate monohydrate [7], and Bis (DL-aspartic acid) sulfate [8] are well studied. Glutamic acid and its ions and salts are called glutamates. The acidic side chain of glutamic acid confers one negative charge under most conditions to proteins, in which this amino acids is found, thus increasing the water solubility of the protein

Aminoguanidine has been one of the interesting species having versatile basicity. A great number of salts of aminoguanidine with different acids have been studied. However, there is no report on aminoguanidinium salts of acidic amino acids in general and "glutamic and aspartic acids" in particular. Hence, our main scope and objectives are to prepare aminoguanidinium salts of amino acids and to characterize them using analytical, IR, and thermal studies, study the mode of thermal decomposition of aminoguanidinium salts, and characterize the antioxidants behavior of aminoguanidinium salts. The present research work has been taken up based on the above objectives and the results are discussed herein.

\section{Experimental section}

Preparation of aminoguanidinium aspartate and glutamate

The aminoguanidinium salts of aspartic acid and glutamic acid were prepared by mixing the respective acid with aminoguanidine bicarbonate in 1:1, 1:2, and 2:1 molar ratios and dissolved in $40 \mathrm{~mL}$ of distilled water. The resulting solutions were heated over water bath and $\mathrm{pH}$ was found to be $6.0,6.79$, and 7.03 , respectively. Then, the volume is reduced to $20 \mathrm{~mL}$ and kept for crystallization at room temperature. After a few days, crystalline solids formed were separated out and washed with ethanol and air dried.

\section{METHODS}

All reagents and chemicals were of $A$. $R$ grade and used without purification. Double-distilled water was used as a solvent for the synthesis and analysis throughout the experiment. Elemental analysis for $\mathrm{C}, \mathrm{H}$, and $\mathrm{N}$ was performed on a Vario-ELIII elemental analyzer. The IR spectra were recorded on a JASCO-4100 spectrophotometer as $\mathrm{KBr}$ pellets in the range of $400-4000 \mathrm{~cm}^{-1}$. The simultaneous TG-DTA studies were under taken on a PerkinElmer SII thermal analyzer and the curves obtained in air using platinum cups as holders with $\sim 3 \mathrm{mg}$ of the samples at the heating rate of $10^{\circ} \mathrm{C} / \mathrm{min}$. 


\section{Antioxidant assays}

Ferric reducing antioxidant power (FRAP) assay

The antioxidant capacities of different salts were estimated according to the procedure described by Pulido et al. [9]. FRAP reagent $(2700 \mu \mathrm{L})$, prepared freshly and incubated at $37^{\circ} \mathrm{C}$, was mixed with appropriate concentration of test sample whose total volume was $360 \mu \mathrm{L}$. A test tube with $360 \mu \mathrm{L}$ of distilled water in place of the sample served as the blank. All the test tubes were incubated at $37^{\circ} \mathrm{C}$ for $30 \mathrm{~min}$ in a water bath. The FRAP reagent was prepared by mixing $2.5 \mathrm{~mL}$ of $20 \mathrm{mM}$ TPTZ in $40 \mathrm{mM} \mathrm{HCl}, 2.5 \mathrm{~mL}$ of $20 \mathrm{mM} \mathrm{FeCl}_{3} 6 \mathrm{H}_{2} \mathrm{O}$, and $25 \mathrm{~mL}$ of $0.3 \mathrm{M}$ acetate buffer (pH-3.6). At the end of incubation, the absorbance of the blue color developed was read immediately at $593 \mathrm{~nm}$ against the reagent blank.

\section{Phosphomolybdenum assay}

The antioxidant activity of samples was evaluated by the green phosphomolybdenum complex formation according to the method of Prieto et al. [10]. Initially, an aliquot of $300 \mu \mathrm{L}$ of samples was taken into a series of test tubes. About $300 \mu \mathrm{L}$ of distilled water taken in a test tube was considered as the blank. All the test tubes were added with $3 \mathrm{~mL}$ of reagent solution $(0.6 \mathrm{M}$ sulfuric acid, $28 \mathrm{mM}$ sodium phosphate, and $4 \mathrm{mM}$ ammonium molybdate) and vortexed well to mix the contents. The mouth of the test tubes was covered with foil and incubated in a water bath at $95^{\circ} \mathrm{C}$ for $90 \mathrm{~min}$. After the samples were cooled to room temperature, the absorbance of the mixture was measured at $695 \mathrm{~nm}$ against the reagent blank. Ascorbic acid (AA) was used as the reference standard and the results were expressed as milligrams of AA equivalents per gram salts.

\section{RESULTS AND DISCUSSION}

The compositions of the salts were fixed by estimating hydrazine moiety in aminoguanidine and the structural abstract is depicted in Scheme 1. The analytical data of the prepared compounds are presented in Table 1 and are in good agreements with the proposed formula. Aspartic acid does not yield the dicationic salt, results viscous solution.

\section{FT-IR spectra}

The important IR bands of the acid, base, and their salts are listed in Table 2 including their band assignments. The FT-IR spectra of free acid, base(Figs. S1-S3), and their salts (Figs. 1-3) are given. The IR spectra show broad band in the region of $3450 \mathrm{~cm}^{-1}$, due to $\mathrm{O}-\mathrm{H}$ stretching. The peaks around 3300 and $1680 \mathrm{~cm}^{-1}$ are assigned to $\mathrm{N}-\mathrm{H}$ and C $=\mathrm{N}$ (imines) stretching vibrations [11]. The bands in the region of 1590-1600 $\mathrm{cm}^{-1}$ and 1390-1350 $\mathrm{cm}^{-1}$ are assigned for asymmetric and symmetric stretching frequencies of the carboxylate ions, respectively [12]. The $\mathrm{N}-\mathrm{N}$ stretching frequency has been observed around $1100 \mathrm{~cm}^{-1}$ which confirms the presence of aminoguanidine moiety.

\section{Thermal studies}

The thermal data of the compounds are listed in Table 3 and the thermograms are shown in Figs. 4-6. The composition of the intermediates and final product is those, which best fit with observed mass loss in the TG curve. The thermogravimetric results are in the good agreement with the DTA data. The thermogram of [Asp(AgunH $\left.)_{2}\right]$ shows two distinct endotherms at 136 and $175^{\circ} \mathrm{C}$ due to the continuous loss of carbon dioxide and two aminoguanidine itself. These results were best fit with observed weight loss. In the preceding step, the amino acid intermediate starts to decompose.

In [Glu(AgunH)], broad endotherm around $170^{\circ} \mathrm{C}$ resulting decarboxylation with the loss of aminoguanidine moiety forming corresponding amino acid, which further decomposes to give gaseous products. The diaminogunidinium salts of glutamic acid show similar decomposition pattern as that of $\left[\operatorname{Asp}(\text { AgunH })_{2}\right]$ around $178^{\circ} \mathrm{C}$ in

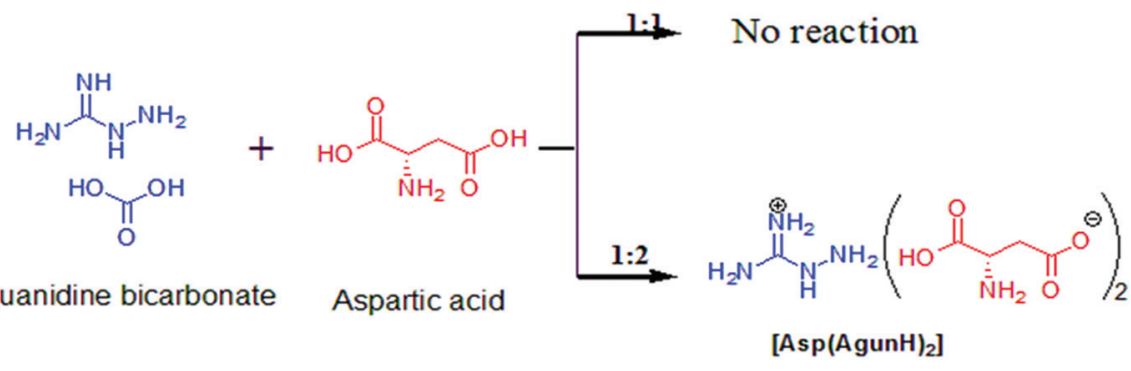

Aminoguanidine bicarbonate Aspartic acid

[Asp(AgunH) $)_{2}$

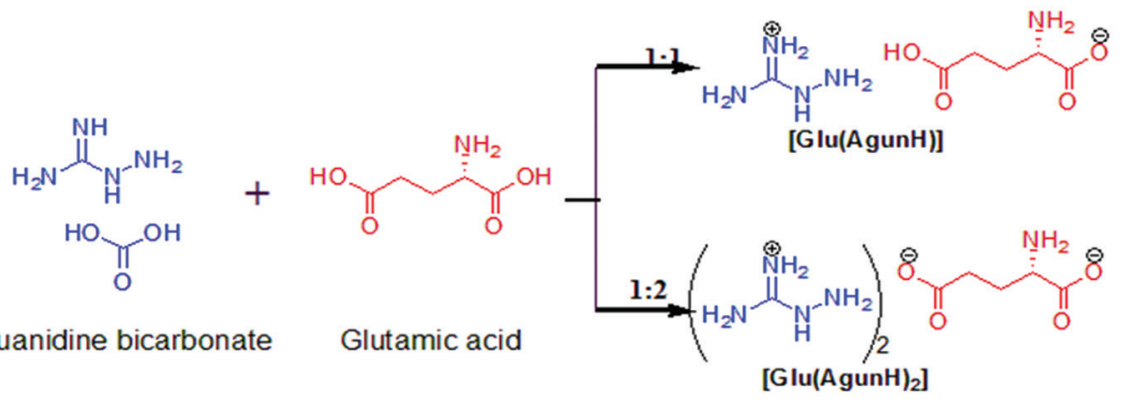

Scheme 1: Synthesis of salts

Table 1: Analytical data

\begin{tabular}{llllll}
\hline Compound & Color & Solubility in water & Melting point $\left({ }^{\circ} \mathbf{C}\right)$ & \multicolumn{2}{c}{ \% of hydrazine } \\
\cline { 4 - 6 } & & & 157 & Observed & Calculated \\
\hline$\left.[\text { Asp(AgunH) })_{2}\right]$ & Orange crystals & Hot water & 171 & 22.97 & 22.85 \\
{$[$ Glu(AgunH) } & White crystals & Cold water & 164 & 14.74 & 14.48 \\
$\left.[\text { Glu(AgunH })_{2}\right]$ & White crystals & Hot water & 21.23 & 21.69 \\
\hline
\end{tabular}


Table 2: FT-IR spectral data (in $\left.\mathrm{cm}^{-1}\right)$

\begin{tabular}{|c|c|c|c|c|c|c|}
\hline Compounds & $v_{\mathrm{O}-\mathrm{H}}$ & $v_{\mathrm{N}-\mathrm{H}}$ & $\mathbf{v}_{\mathrm{C}=\mathrm{N} \text { (iminidine) }}$ & $\begin{array}{l}v_{\text {oco }} \\
\text { asym }\end{array}$ & $\begin{array}{l}v_{\text {oco }} \\
\text { sym }\end{array}$ & $v_{\mathrm{N}-\mathrm{N}}$ \\
\hline$($ AgunH $) \mathrm{HCO}_{3}$ & - & 3295 & 1660 & - & - & 1113 \\
\hline Aspartic acid & 3410 & 3283 & - & 1706 & - & - \\
\hline$\left[\right.$ Asp $\left.(\text { AgunH })_{2}\right]$ & 3440 & 3230 & 1680 & 1634 & 1352 & 1114 \\
\hline Glutamic acid & 3270 & 3200 & - & 1700 & - & - \\
\hline [Glu(AgunH)] & 3470 & 3250 & 1673 & 1590 & 1395 & 1146 \\
\hline$\left[\mathrm{Glu}(\text { AgunH })_{2}\right]$ & 3450 & 3335 & 1670 & 1630 & 1353 & 1113 \\
\hline
\end{tabular}

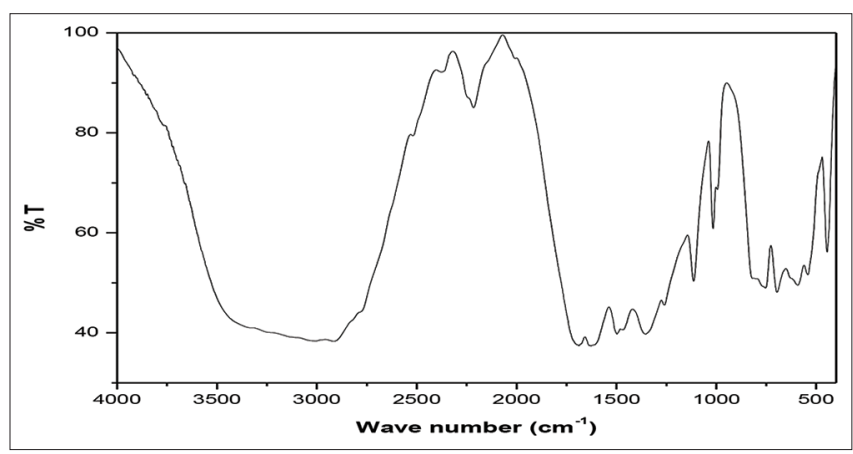

Fig. S1: IR Spectrum of Aminoguanidine bicarbonate

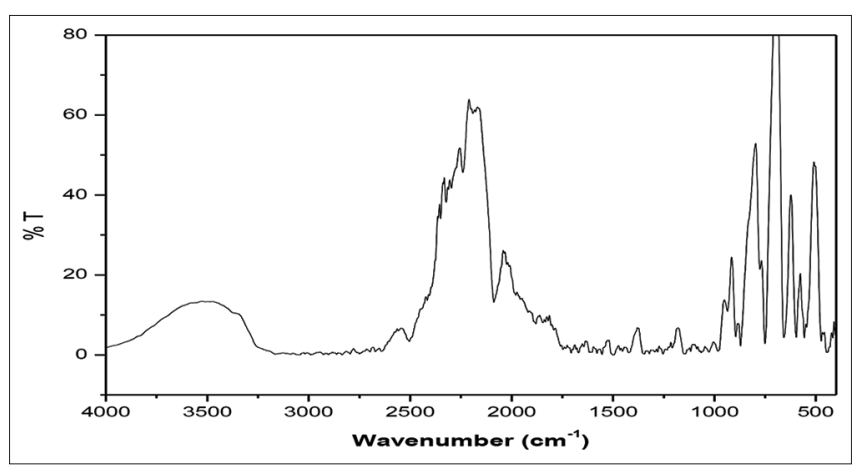

Fig. S2: IR Spectrum of Aspartic acid

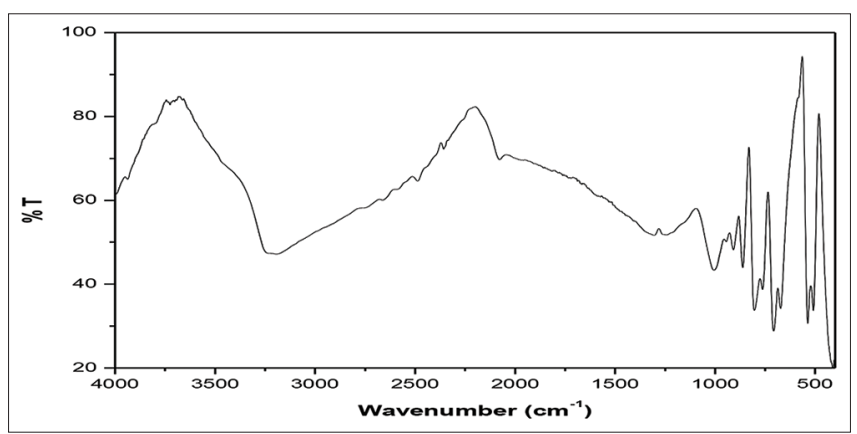

Fig. S3: IR Spectrum of Glutamic acid

DTA. Then, the remaining $35 \%$ of mass loss occur exothermally in the temperature range of $550-700^{\circ} \mathrm{C}$

\section{Antioxidant studies}

There are many in vitro methods to assess the antioxidant activity and depend on various generation of free radicals acting through different mechanisms to cover all aspects of antioxidant efficacy. Free radical scavenging activity and reducing capacities of newly synthesized

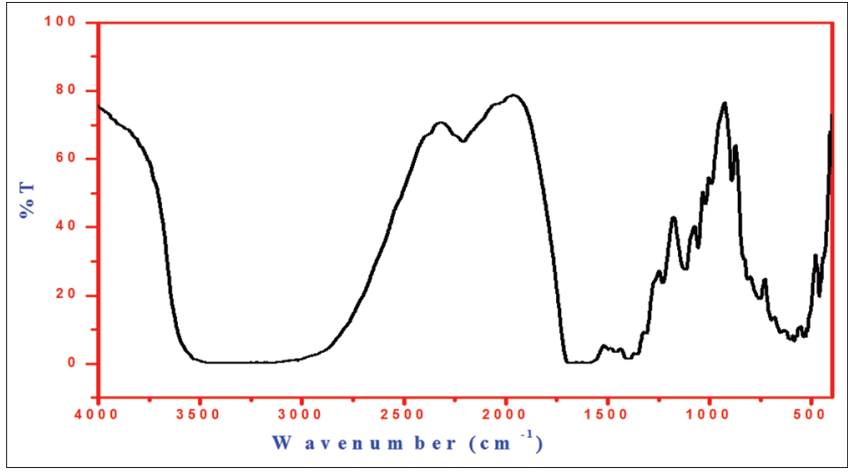

Fig. 1: FT-IR spectrum of $\left[\operatorname{Asp}(\operatorname{AgunH})_{2}\right]$

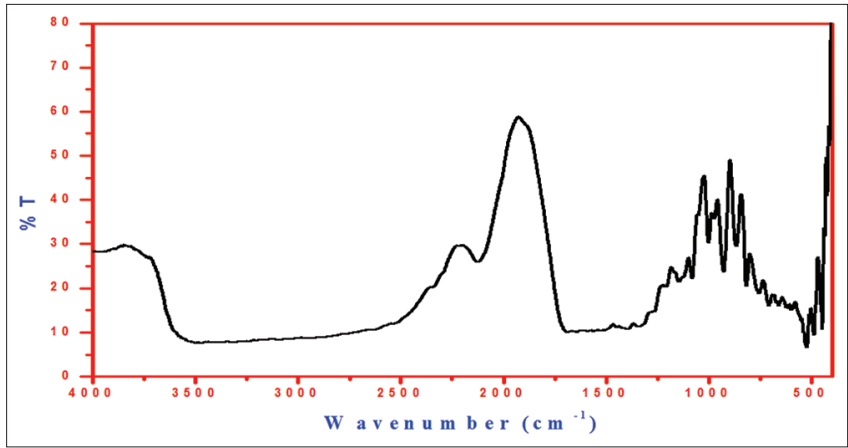

Fig. 2: FT-IR spectrum of [Glu(AgunH)]

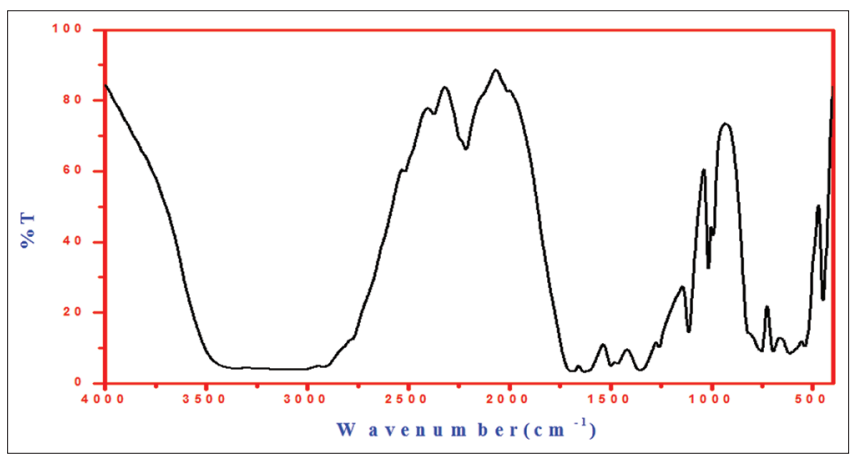

Fig. 3: FT-IR spectrum of [Glu(AgunH $\left.)_{2}\right]$

compounds were confirmed by FRAP and phosphomolybdenum assay. The salts exhibit good antioxidant property is due to the presence of nitrogen content [13].

\section{FRAP assay}

FRAP assay is an inexpensive procedure which is simple, reproducible, and rapid that measures the ability of antioxidant compound to reduce the ferric ion $\mathrm{Fe}^{3+}$ to ferrous ion $\mathrm{Fe}^{2+}$, as a measure of total antioxidant capacity. The scavenging activities are expressed as $50 \%$ inhibitory concentration $\left(\mathrm{IC}_{50}\right)$ values, which represent the concentrations of the compound used to scavenge $50 \%$ free radicals. The $\mathrm{IC}_{50}$ values for compounds and standard (natural AA) against free radicals are tabulated in Table 4.

Among the three salts, $\left[\mathrm{Glu}(\mathrm{AgunH})_{2}\right]$ has the highest antioxidant property comparable with that of standard AA. Our results showed significant ferric reducing power which indicated the hydrogendonating ability of the extract. 
Table 3: Thermal data

\begin{tabular}{|c|c|c|c|c|c|}
\hline \multirow[t]{3}{*}{ Compounds } & \multirow{3}{*}{$\begin{array}{l}\text { DTA } \\
\text { Peak temp }\left({ }^{\circ} \mathrm{C}\right)\end{array}$} & \multicolumn{3}{|c|}{ Thermogravimetry } & \multirow[t]{3}{*}{ Intermediates/end product } \\
\hline & & \multirow[t]{2}{*}{ Temp. range $\left({ }^{\circ} \mathrm{C}\right)$} & \multicolumn{2}{|c|}{ Mass loss \% } & \\
\hline & & & Observed & Calculated & \\
\hline \multirow[t]{3}{*}{$\left(\right.$ AgunH) $\mathrm{HCO}_{3}$} & $(+) 135$ & $90-180$ & 68.50 & 69.00 & $\mathrm{NH}=\mathrm{C}=\mathrm{NH}$ \\
\hline & $(+) 175$ & & & & \\
\hline & $(-) 320$ & $180-600$ & 100.00 & 100.00 & Complete decomposition \\
\hline \multirow{3}{*}[\operatorname{Asp}(\operatorname{AgunH})_{2}]{} & $(+) 136$ & $100-200$ & 70.00 & 69.28 & Elimination of $2 \mathrm{Agun}$ and $\mathrm{CO}_{2}$ \\
\hline & $(+) 175$ & & & & \\
\hline & $(-) 634$ & $200-700$ & 100 & 100 & Complete decomposition \\
\hline \multirow[t]{2}{*}{ [Glu(AgunH)] } & $(+) 174$ & $120-231$ & 54.00 & 53.39 & Elimination of Agun and $\mathrm{CO}_{2}$ \\
\hline & $(-) 611$ & $231-700$ & 100 & 100 & Complete decomposition \\
\hline \multirow[t]{2}{*}[\operatorname{Glu}(\operatorname{AgunH})_{2}]{} & $(+) 178$ & $110.6-236$ & 65.4 & 65.76 & Elimination of $2 \mathrm{Agun}$ and $\mathrm{CO}_{2}$ \\
\hline & $(-) 610$ & $236-700$ & 100 & 100 & Complete decomposition \\
\hline
\end{tabular}

(+): Endo, (-): Exo

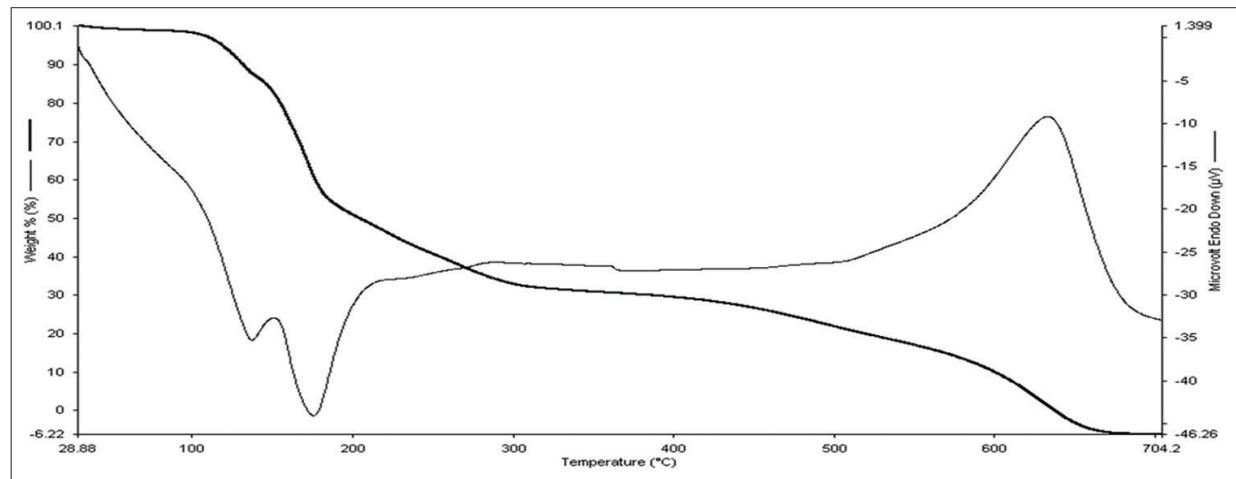

Fig. 4: Simultaneous TG - DTA of [Asp(AgunH) $\left.)_{2}\right]$

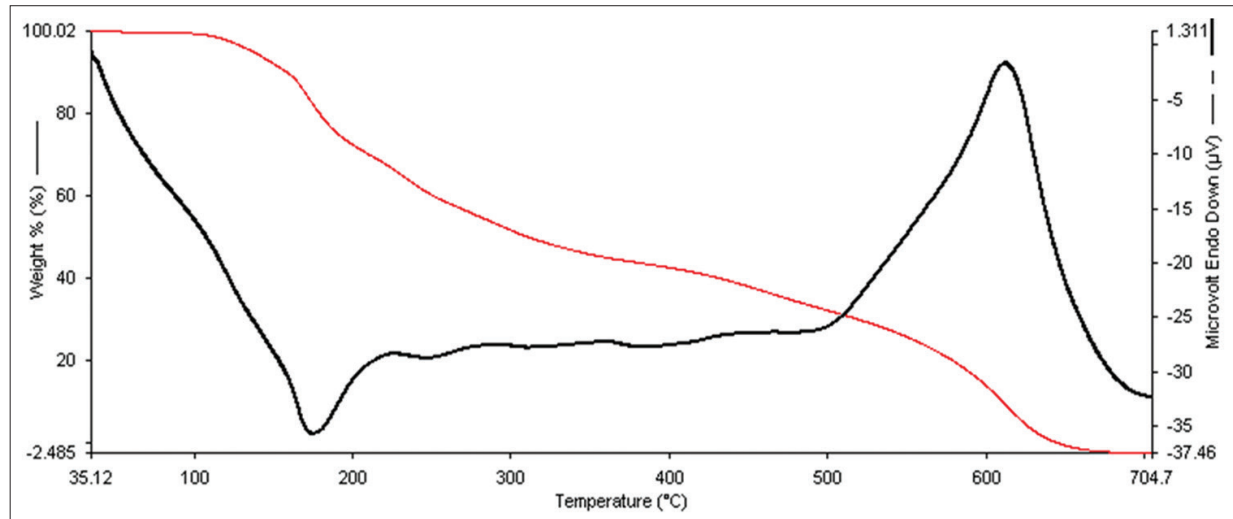

Fig. 5: Simultaneous TG - DTA of [Glu(AgunH)]

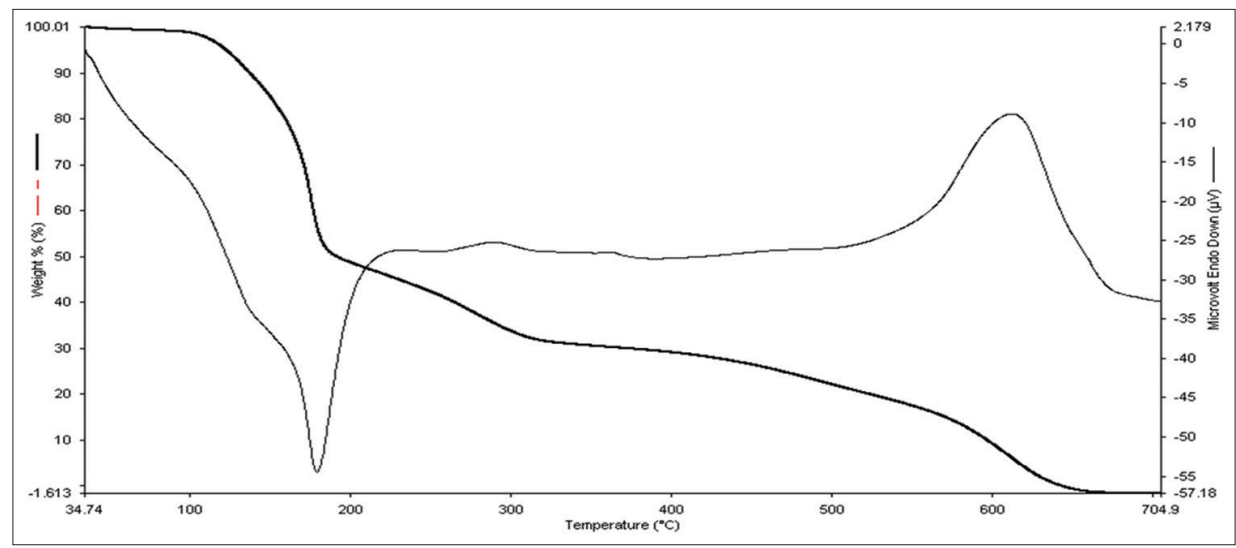

Fig. 6: Simultaneous TG - DTA of [Glu(AgunH) $\left.{ }_{2}\right]$ 
Table 4: Antioxidant activities of compounds and Vitamin C against radicals

\begin{tabular}{lll}
\hline Compounds & $\mathbf{I C}_{\mathbf{5 0}}(\boldsymbol{\mu M})$ & \\
\cline { 2 - 3 } & $\begin{array}{l}\text { Ferric reducing } \\
\text { antioxidant power }\end{array}$ & Phosphomolybdenum \\
\hline$\left[\right.$ Asp $\left.(\text { AgunH })_{2}\right]$ & 43.98 & 108.89 \\
{$[\mathrm{Glu}($ AgunH $)]$} & 58.01 & 90.18 \\
{$\left[\mathrm{Glu}(\text { AgunH })_{2}\right]$} & 50.50 & 73.10 \\
Vitamin C & 52.45 & 75.59 \\
\hline
\end{tabular}

Phosphomolybdenum assay

Phospomolybdenum method is alternative to the methods available for the evaluation of antioxidant capacities due to its simplicity. This method is based on the reduction of phosphomolybdic acid to phosphomolybdenum blue complex by sodium sulfide. The obtained complex is oxidized by addition of nitrite and this causes a reduction in intensity.

\section{CONCLUSION}

The new aminoguanidinium salts of aspartic acid and glutamic acid have been prepared by the reaction of aqueous solution containing aminoguanidine bicarbonate with acidic amino acids. Aspartic acid forms bis-aminoguanidinium salt, whereas glutamic acid forms both mono- and bis-aminogunidinium salts. The IR spectral data of the aminogunidinium salts of aforesaid acids show $\mathrm{N}-\mathrm{N}$ stretching frequencies in the region of 1202-1110 $\mathrm{cm}^{-1}$ revealing the presence of aminoguanidinium moiety. The asymmetric and symmetric stretching frequencies of carboxylates are seen in the region of 1680-1670 and 1395-1352 cm 1. The thermal studies of the salts revealed that all of them decompose in endo, followed by exothermic fashion, giving gaseous end products. The antioxidant properties of these salts were studied using FRAP and phosphomolybdenum assay. Results showed significant ferric reducing power which indicated the hydrogen-donating ability of the extract.

\section{ACKNOWLEDGMENTS/FUNDING}

The author is gratefully acknowledges the financial assistance received from the Department of Science and Technology, New Delhi, India (Grant No. SR/WOS[A]/CS-42/2013).

\section{AUTHORS' CONTRIBUTIONS}

The study was designed and executed by S. Parveen and Dr. S. Govindarajan. All the experimental section and analysis section were performed by them also. Editing and reviewing were done by Dr. D. Manikandan and Dr. P. A. Periasamy.

\section{CONFLICTS OF INTEREST}

There are no conflicts to declare.

\section{REFERENCES}

1. Manikandan D, Jegadeeshwari LA, Gandhi NN. Biological activity sources from traditionally usedtribe and herbal plants material. Asian J Pharm Clin Res 2015;8:11-2.

2. Manikandan D, Prakash DG, Arun J, Gandhi NN, Mani U, Kathiravan K. Antibacterial and anticancer activities of silver nanoparticles biosynthesized using Embelia ribes Burm. f. Berries extract. Indian J Exp Biol 2019;57:175-80.

3. Manikandan D, Denison MI, Manikandan A, Gandhi NN, Kathiravan K, Baykal A. Biogenic synthesis, characterization of gold and silver nanoparticles from Coleus forskohlii and their clinical importance. J Photochem Photobiol B 2018;183:251-7.

4. Klussmann M, Izumi T, White AJ, Armstrong A, Black-Mond DG. Emergence of solution-phase homochirality via crystal engineering of amino acids. J Am Chem Soc 2007;129:7657-60.

5. Derissen JL, Endeman HJ, Peerdeman AF. The crystal and molecular structure of L-aspartic acid. Acta Cryst 1968;1324:1349-54.

6. Bendeif $\mathrm{E}$, Jelsch $\mathrm{C}$. The experimental library multipolar atom model refinement of L-aspartic acid. Acta Cryst 2007;C63:361-4.

7. Sridhar B, Srinivasan N, Rajaram RK. L-aspartic acid nitrate-L-aspartic acid (1/1). Acta Cryst 2002;E58:1372-4.

8. Srinivasan N, Sridhar B, Rajaram RK. L-aspartic acid nitrate-L-aspartic ACID. Acta Cryst 2001;E57:679-81

9. Pulido R, Bravo L, Sauro-Calixto F. Antioxidant activity of dietary polyphenols as determined by a modified ferric reducing/antioxidant power assay. Agric Food Chem 2000;48:3396-402.

10. Prieto P, Pineda M, Aguilar M. Spectrophotometric quantitation of antioxidant capacity through the formation of a phosphomolybdenum complex: Specific application to the determination of Vitamin E. Anal Biochem 1999;269:337-41

11. Parveen S, Nguyen HH, Govindarajan S. Synthesis, crystal structure, molecular docking and cytotoxicity of zwitterionic 3-(4-amino-3imino-5-oxo-2,3,4,5-tetrahydro-[1,2,4]triazin-6-yl)-propionic acid. J Indian Chem Soc 2018;95:651-9.

12. Parveen S, Govindarajan S, Puschmann H, Revathi R. Synthesis, crystal structure and biological studies of new hydrazone ligand, 2-(methoxycarbonyl-hydrazono)-pentanedioic acid and its silver(I) complex. Inorganica Chim Acta 2018;477:66-74.

13. Parveen S, Velmurugan G, Sinn E, Venuvanalingam P, Govindarajan S. Water-soluble cobalt(II) and cobalt(III) complexes supported by new triazine Schiff base ligands: Synthesis, structure and biological evaluation. J Photochem Photobiol 2018;B189:152-64. 